\title{
Functional segregation of the human basal forebrain using resting state neuroimaging
}

\author{
Ross D. Markello ${ }^{1,2}$, R. Nathan Spreng ${ }^{1,2}$, Wen-Ming Luh ${ }^{3}$, Adam K. Anderson ${ }^{1,2}$, and Eve \\ De Rosa ${ }^{1,2}$ \\ ${ }^{1}$ Department of Human Development, Cornell University, Ithaca, NY, 14853 \\ ${ }^{2}$ Human Neuroscience Institute, Cornell University, Ithaca, NY, 14853 \\ ${ }^{3}$ Cornell Magnetic Resonance Imaging Facility, Cornell University, Ithaca, NY, 14853
}

\begin{abstract}
The basal forebrain (BF) is poised to play an important neuromodulatory role in brain regions important to cognition due to its broad projections and complex neurochemistry. While significant in vivo work has been done to elaborate BF function in nonhuman rodents and primates, comparatively limited work has examined the in vivo function of the human BF. In the current study we used multi-echo resting state functional magnetic resonance imaging (rs-fMRI) from 100 young adults (18-34 years) to assess the potential segregation of human BF nuclei as well as their associated projections. Bottom-up clustering of voxel-wise functional connectivity maps yielded adjacent functional clusters within the BF that closely aligned with the distinct, hypothesized nuclei important to cognition: the nucleus basalis of Meynert (NBM) and the medial septum/diagonal band of Broca (MS/DB). Examining their separate functional connections, the NBM and MS/DB revealed distinct projection patterns, suggesting a conservation of nucleispecific functional connectivity with homologous regions known to be anatomically innervated by the BF. Specifically, the NBM demonstrated coupling with a widespread cortical network as well as the amygdala, whereas the MS/DB revealed coupling with a more circumscribed network, including the orbitofrontal cortex and hippocampal complex. Collectively, these in vivo rs-fMRI data demonstrate that the human BF nuclei support functional networks distinct aspects of resting-state functional networks, suggesting the human BF may be a neuromodulatory hub important for orchestrating network dynamics.
\end{abstract}

Keywords: resting state functional MRI, basal forebrain, multi-echo, functional networks

\section{Highlights}

- The basal forebrain NBM and the MS/DB support two distinct functional networks

- Functional networks closely overlap with known anatomical basal forebrain

- Basal forebrain networks are distinct from known resting-state functional networks

\section{Introduction}

The basal forebrain (BF) serves as a major neuromodulatory hub for brain areas critical to cognition (Rye et al., 1984; Wenk, 1997; Yang et al., 2017). Due to its widespread projections (Descarries et al., 2004; Lysakowski et al., 1989; Mesulam et al., 1986), the BF has the capacity to play an important modulatory role in a range of neural dynamics (Baxter and Bucci, 2013; Hasselmo and 
Sarter, 2011; Ovsepian et al., 2016), influencing sensory plasticity (Picciotto et al., 2012; Rasmusson, 2000; Sarter and Bruno, 1997; Shinoe et al., 2005), attention (Botly and De Rosa, 2007, 2008, 2009, 2012; Gritton et al., 2016; Ljubojevic et al., 2014; Parikh et al., 2007), and memory (Chudasama et al. 2004; Croxson et al. 2011; cf. Baxter et al. 1995).

BF function has also been implicated in the cognitive changes associated with normal and pathological aging (Coyle et al., 1983; Davies and Maloney, 1976; Grothe et al., 2013; Muir, 1997; Perry et al., 1977; Whitehouse et al., 1982), and degeneration of the BF and its anatomical projections is hypothesized to have both functional and cognitive implications across the lifespan (Pepeu and Giovannini, 2017; Schmitz et al., 2016). A better understanding of human BF functional connections may provide insight into the distinct capacity of the BF to modulate the cortical and subcortical interactions that support cognition (Bell and Shine, 2016; Muñoz and Rudy, 2014).

Noninvasive assessments of human BF function have proven challenging due to difficulties in localization with functional magnetic resonance imaging (fMRI), especially when using whole-brain measurements. A stereotaxic probabilistic map of the BF generated using postmortem brains (Zaborszky et al., 2008) has partially ameliorated these issues (Li et al., 2014; Schmitz et al., 2016; Zhang et al., 2016). However, while significant work has demonstrated divisions in the neuromodulatory projections of the BF in nonhuman animals with increasing specificity, to date no work has examined how the BF may functionally differentiate in humans (Golden et al., 2016; Hedreen et al., 1984; Kim et al., 2016, 2015; Kondo and Zaborszky, 2016; Ma and Luo, 2012; Mesulam et al., 1983b; Swanson and Cowan, 1979). It is thus important to assess the human functional networks mediated by the $\mathrm{BF}$ as it not only relates to existing knowledge of anatomical projections derived from these nonhuman animals investigations, but also to our current understanding of functional connections revealed through resting state fMRI data.

Rodent and nonhuman primate literature has shown the BF comprises distinct subdivisions, termed Ch1-4, delineating the BF at both a cellular and functional level (Mesulam et al., 1983b). The Ch1-3 groups, termed the medial septum and diagonal band of Broca (MS/DB), have projections that extend primarily into the hippocampal complex and hypothalamus (Hedreen et al., 1984; Swanson and Cowan, 1979), while the Ch4 group, termed the nucleus basalis of Meynert (NBM; also includes the substantia innominata), provides innervation to the entire cortical mantle and amygdala (Hedreen et al., 1984; Mesulam et al., 1983b). These subdivisons were originally differentiated based on their cholinergic projections (Mesulam et al., 1983b), but later work has demonstrated that the majority of projections from these regions are GABA- (Brashear et al., 1986; Gritti et al., 1993) and glutamatergic (Gritti et al., 2006). Indeed, co-release of GABA and glutamate from Ch1-4 projections is common (Gritti et al., 2006; Manns et al., 2001; Saunders et al., 2015), and recent work has shown that cholinergic and non-cholinergic projections from these BF functional subdivisions may work in tandem to influence cognition (Kim et al., 2015).

Thus, given the breadth and heterogeneity of its neuromodulatory connections, assessing how the BF relates to functional connectivity would be especially beneficial. Understanding how these neuromodulatory systems may influence functional connections and help inform whole-brain communication patterns is an especially relevant, yet understudied, aspect of the human fMRI literature (Bell and Shine, 2016; Shine et al., 2017; Spronk et al., 2017). The current study aimed to investigate the functional profiles of the BF using multi-echo resting-state fMRI (rsfMRI) data in young adults. Resting-state fMRI, in particular, has shown to be useful for delineating functional networks relevant to cognition (Stevens and Spreng, 2014), and recent developments in multi-echo fMRI have been shown to better separate BOLD responses tied to neural activity from non-BOLD artifacts (Kundu et al., 2013, 2017). We thus took advantage of a multi-echo approach to potentially distinguish activity within BF nuclei, along with their functional connectivity to known targets. We further compared traditional single-echo analyses with multi-echo techniques to assess the pro- 
portional dissociation of the MS/DB from the NBM. We hypothesized that rsfMRI would reveal functional networks reflecting known delineations of the BF, in support of cross-species homology previously demonstrated with ex vivo techniques (Emre et al., 1993; Mesulam et al., 1988; Saper and Chelimsky, 1984; Selden et al., 1998).

\section{Materials and Methods}

\subsection{Participants}

Participants were 100 young adults (18-34 years, mean $=22.6 \pm 3.2$ years, 58 females) with normal or corrected-to-normal vision and no history of psychiatric or neurological illness. All participants gave informed consent in accordance with the Institutional Review Board at Cornell University.

\subsection{MRI data collection}

Imaging data were acquired on a 3T GE Discovery MR750 MRI scanner with a 32-channel head coil at the Cornell Magnetic Resonance Imaging Facility in Ithaca, NY. Anatomical scans were captured using a T1-weighted volumetric MP-RAGE sequence $(\mathrm{TR}=7.7 \mathrm{~ms}$; TE $=3.42 \mathrm{~ms}$; $7^{\circ}$ flip angle; $1.0 \mathrm{~mm}$ isotropic voxels, 176 slices). Two 10 minute and 12 second BOLD resting state functional scans were acquired using a multi-echo EPI pulse sequence $\left(\mathrm{TR}=3000 \mathrm{~ms}\right.$; $\mathrm{TE}_{1}$ $=13.7 \mathrm{~ms}, \mathrm{TE}_{2}=30.0 \mathrm{~ms}, \mathrm{TE}_{3}=47.0 \mathrm{~ms} ; 83^{\circ}$ flip angle; $3.0 \mathrm{~mm}$ isotropic voxels; 46 slices). Participants were instructed to remain awake with their eyes open for the duration of both functional scans.

\subsection{MRI data preprocessing}

All fMRI data were preprocessed in AFNI (version 16.0.12; Cox 1996), with the exception of skull-stripping and segmentation, which were done with FSL's Brain Extraction Tool (bet2; Jenkinson et al. 2005; Smith 2002) and FMRIB's Automated Segmentation Tool (fast; Zhang et al. 2001), respectively. Analysis was conducted using a combination of AFNI and Python (v3.5.3; https://www.python.org/) with associated NumPy (v1.11.1; Walt et al. 2011) and SciPy (v0.17.1; Jones et al. 2001) modules.

\subsubsection{Multi-echo preprocessing}

Multi-echo data were processed using ME-ICA (meica.py, version 2.5 beta 8; Kundu et al. 2012). The ME-ICA preprocessing pipeline was as follows: (1) slice timing correction was applied to all the TE series; (2) the first echo series $\left(\mathrm{TE}_{1}\right)$ was aligned to the first time point using a 6-parameter rigid-body alignment procedure to generate motion correction parameters; (3) all TE series were used to generate a $\mathrm{T} 2^{*}$ map of the brain, which was co-registered to the skull-stripped anatomical to generate co-registration parameters; (4) the skull-stripped anatomical was linearly warped to MNI (Montreal Neurological Institute) space with the single-subject reference brain (Collins et al., 1994; Holmes et al., 1998) using a 12-parameter affine alignment procedure; (5) a brain mask was created from the mean image of the first echo $\left(\mathrm{TE}_{1}\right)$ and applied to all the echoes; (6) motion correction, co-registration, and normalization warping parameters were simultaneously applied to each TE series; and (7) TE series were resampled to $2 \mathrm{~mm}$ isotropic voxels.

The three TE series were then optimally combined to form a single, weighted time series. This combined time series was subjected to a FastICA decomposition and component sorting method (described in Kundu et al. 2013). Briefly, T2* signal in fMRI data linearly increases with TE, permitting classification of components as BOLD $(\mathrm{T} 2 *)$ or non-BOLD (S0) based on their adherence to a TE-dependent signal increase across the multiple echoes (see Kundu et al. 2017 for a recent 

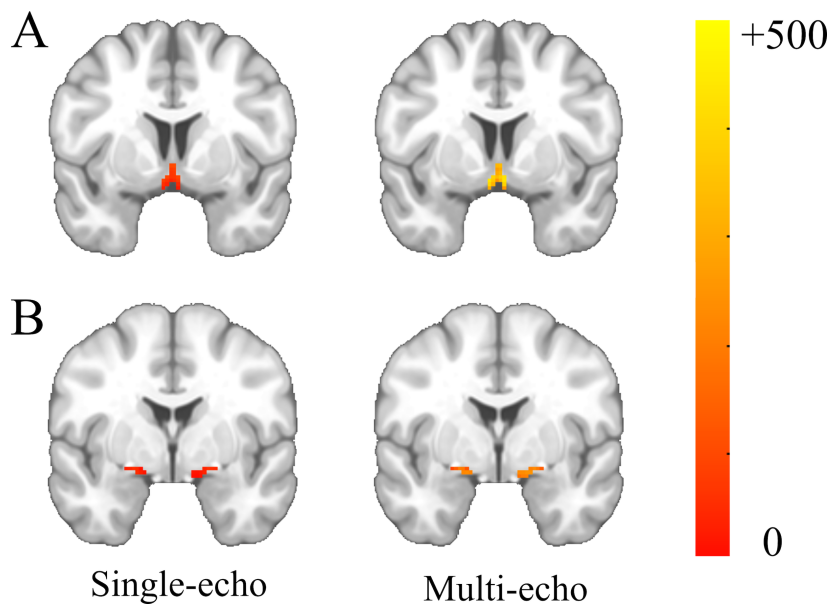

Figure 1. Temporal signal-to-noise ratio. A. The medial septum/diagonal band of Broca, and B. the nucleus basalis of Meynert for both single- and multi-echo data. Temporal signal-to-noise was calculated as the absolute value of the mean divided by the standard deviation of the time series, as in Equation 1. Figure made with FSLeyes software.

review). Components classified as $\mathrm{T} 2 *$ BOLD were recombined to create a denoised time series. Multi-echo processed data were not smoothed further.

\subsubsection{Single-echo preprocessing}

To compare the efficacy of ME-ICA in assessing functional connectivity, we separately preprocessed the middle echo image (i.e., $\mathrm{TE}_{2}$ ). The middle echo image was carried through the same processing pipeline detailed above through the end of step 7 , at which point it was processed in a manner similar to Power et al. (2014).

Nuisance variables were generated by combining motion estimates (6), their derivatives (6), and tissue-specific estimates generated by averaging signal across voxels in tissue-specific masks (produced by FSL's fast). In all, six tissue-specific estimates were used: cerebrospinal fluid (CSF), white matter (WM), and the global signal (whole brain), as well as their derivatives.

Frame-wise displacement (FD) and change in signal variance (DVARS, Derivative of signal VARiance) traces were calculated from the middle echo time series using tools implemented in Nipype (Gorgolewski et al., 2011); visual inspection of these traces across subjects suggested that $\mathrm{FD} \geq 0.2 \mathrm{~mm}$ and DVARS $\geq 1.6 \% \triangle \mathrm{BOLD}$ were adequate cutoffs for identifying abnormal motion. Following Power et al. (2012), temporal masks were created from each of these traces: frames that exceeded the threshold were marked for removal, along with the frame before and the two frames after the marked timepoint. The intersection of the temporal masks generated from each trace were used to create a final mask.

The final temporal mask was applied to all nuisance variables and the $\mathrm{TE}_{2}$ time series; standardized and detrended nuisance variables were regressed against the time series, generating least-squares fit beta maps. Beta maps were multiplied with the "full" (i.e., unmasked) nuisance variables to generate a modeled time-series, which was subtracted from the observed time series. The resultant residual time series was quadratically detrended, bandpass filtered (0.009-0.08 Hz) and smoothed (6mm FWHM) inside a whole brain mask (generated in step 5 of multi-echo preprocessing, above). Finally, the temporal mask was reapplied to the smoothed data; the first and last 13 TRs of each 
run were also removed to account for temporal smoothing due to filtering.

\subsection{Regions of interest}

Regions of interest (ROIs) were generated from the BF probabilistic maps in the SPM Anatomy toolbox (Eickhoff et al., 2007; Zaborszky et al., 2008). A whole BF mask was created by combining the probability masks, resampling to functional space, and thresholding at $>40 \%$ probability. Individual ROI masks were created via the same procedure using the separate probability masks. Resultant masks were 95 voxels in size for entire BF (Ch1-4, BF), 37 voxels for MS/DB (Ch1-3), and 51 voxels for NBM (Ch4).

\subsubsection{Basal forebrain $\mathrm{T}^{*}$ values}

The $\mathrm{T}^{*}$ value of each $\mathrm{BF}$ nucleus was calculated using the map generated during step (3) of the multi-echo data processing pipeline. Values were averaged within each ROI prior to being averaged across the two resting states runs for each individual. Resultant values were averaged across individuals to generate optimal $\mathrm{T}^{*}$ values for the NBM $(29.97 \mathrm{~ms} \pm 4.48)$ and the MS/DB $(28.63 \mathrm{~ms} \pm 9.94)$. The standard deviation of $\mathrm{T} 2 *$ values were also calculated for within the NBM $(9.02 \mathrm{~ms} \pm 1.71)$ and within the MS/DB $(10.63 \mathrm{~ms} \pm 2.62)$, suggesting some inhomogeneity within ROIs. In general, $2^{*}$ values for both regions seem to align with echo times traditionally used in resting-state functional analyses.

\subsubsection{Quality assessment}

Previous work has demonstrated the efficacy of ME-ICA in removing physiological and motionrelated noise (Dipasquale et al., 2017; Kundu et al., 2015) and increasing temporal SNR (DuPre et al., 2016). However, given the proximity of the BF to regions which typically experience signal dropout and distortion, we wanted to assess the relative tSNR of each ROI to ensure adequate ability to detect effects of interest. Using AFNI's 3dTstat -cvarinvNOD, tSNR was calculated for both multi- and single-echo preprocessed data inside each ROI for individual subjects according to:

$$
t S N R=\frac{\left|\mu_{x y z}\right|}{\sigma_{x y z}}
$$

where $\mu_{x y z}$ is the average signal within a given voxel and $\sigma_{x y z}$ is the standard deviation.

Subject-level tSNR values were averaged to generate an approximate group-level tSNR metric (Figure 1) for the NBM (multi-echo: $254.97 \pm 129.01$, single-echo: $68.01 \pm 49.43$ ) and MS/DB (multi-echo: $380.48 \pm 149.44$, single-echo: $111.28 \pm 46.56$ ). Given the use of global signal regression on single-echo data during preprocessing, tSNR for single-echo data was calculated on a more minimally preprocessed $\mathrm{TE}_{2}$ time series following conventional denoising performed in Kundu et al. (2013). Calculation of tSNR for multi-echo data was performed using the time series generated by projecting the BOLD-selected components into the time domain (referred to as the high- $\kappa$ time series, Kundu et al. 2013).

\subsection{Functional connectivity}

Multi-echo independent components regression (ME-ICR; Kundu et al. 2013) was used to assess functional correlations for multi-echo data; ME-ICR has been demonstrated to reliably capture known connections at the single-subject level with single-voxel coordinates (Kundu et al., 2013). Traditional voxel-wise, time-series correlations were used to assess functional relationships for singleecho data. 
A
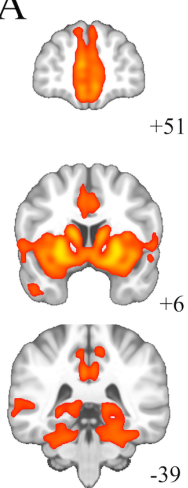

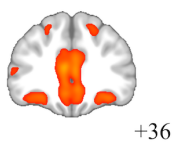

$+3$
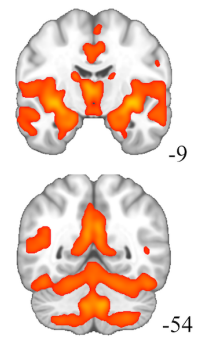
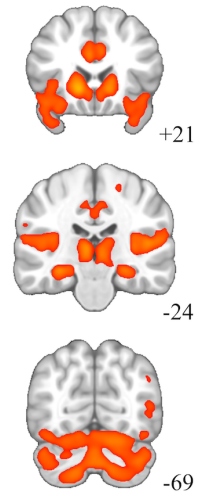

B
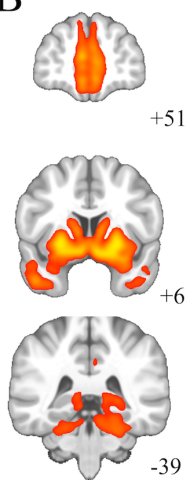

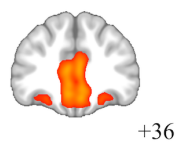

$+36$
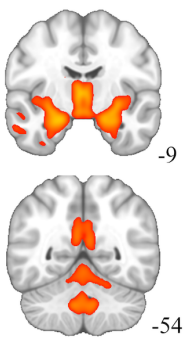
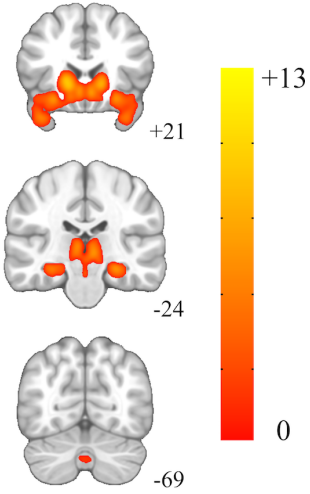

Figure 2. Functional correlations of the basal forebrain (BF, Ch1-4). A. Multi-echo preprocessed and B. single-echo preprocessed data. Significant connections are presented at a $\mathrm{p} \leq 0.001$ threshold, cluster-corrected to alpha $\leq 0.01$, and presented as Z-scores. Results are presented on the MNI-152 reference image to aid in anatomical localization.

\subsubsection{Multi-echo rsFC}

Rather than using the BOLD time series, weights for voxels in each ROI were extracted from the independent BOLD components identified during the ME-ICA component sorting (described above) to ensure correlated activity represented BOLD-specific functional coupling. The Pearson correlation was calculated between the extracted vector of weights and that of every other voxel in the brain. Correlation maps were converted to $\mathrm{Z}$ scores using Fisher's $r$-to- $Z$ transform:

$$
Z=\operatorname{arctanh}(r) * \sqrt{d f-3}
$$

where $d f$ is the degrees of freedom - in this case, the number of BOLD components (i.e., the length of the weight vector), which varied across individuals and sessions. Z-score maps were averaged across both functional scans for a given subject before being brought to a group-level analysis.

We conducted separate, one-sample t-tests for the whole BF, NBM, and MS/DB Z-score maps. Group analyses were confined to a gray matter mask derived using FSL's fast on the single-subject MNI reference template resampled to functional space, and group results were smoothed with a $6 \mathrm{~mm}$ FWHM Gaussian kernel.

\subsubsection{Single-echo rsFC}

Signal for voxels in each ROI were extracted and averaged, then correlated against every voxel in the brain. Correlation maps were converted to Z-score using Equation 2. Degrees of freedom were calculated with:

$$
d f=\left(N_{T R}-N_{\text {reg }}\right) * f_{\text {remain }}
$$

where $N_{T R}$ is the number of TRs that remained after the final temporal mask was applied, $N_{\text {reg }}$ is the number of nuisance regressors (here, 18 ), and $f_{\text {remain }}$ is the proportion of frequencies remaining after bandpass filtering (here, 0.426), as in Kundu et al. (2013) and Power et al. (2014). Group level analyses were performed on resultant Z-score maps as described for multi-echo data.

Only positive findings are presented for single-echo results due to the potential introduction of artifactual anti-correlations through the use of global signal regressions (Murphy et al., 2009; Saad 

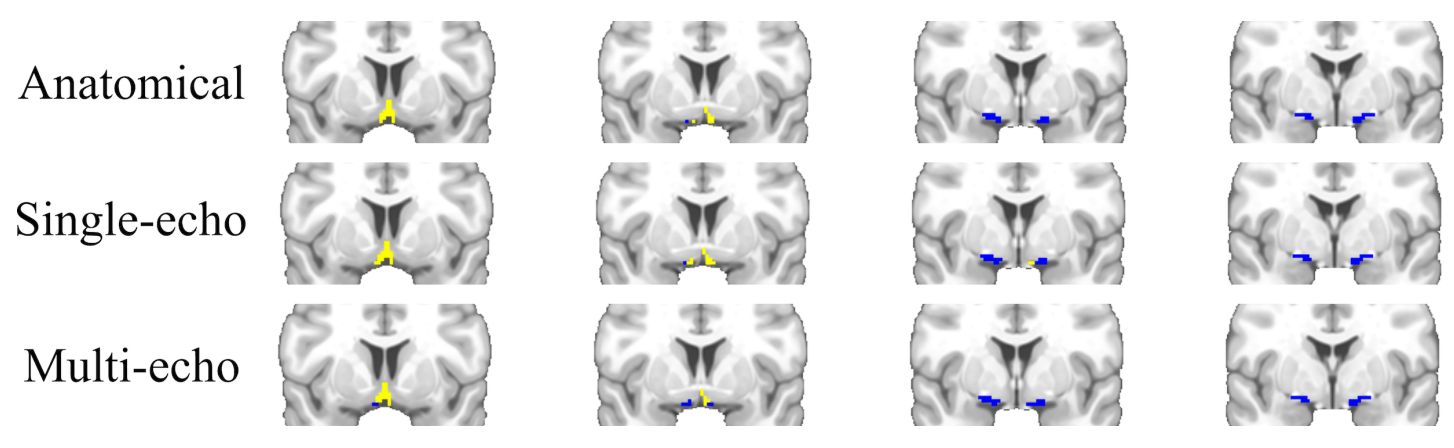

Figure 3. Anatomical and functional clusters of the basal forebrain. Top. Anatomical BF region of interest generated from Zaborszky et al. (2008) probability maps. Center. Cluster solution from ME-ICR functional connectivity data. Bottom. Cluster solution from single-echo functional connectivity data. Yellow corresponds to c1, blue to c2 in Table 1.

et al., 2012; Van Dijk et al., 2010; Weissenbacher et al., 2009). ME-ICR has been shown to avoid the skewed distribution of correlation coefficients found in traditional connectivity analyses (Kundu et al., 2013), thus supplanting the need for additional corrective measures with multi-echo data.

\subsection{Ward clustering}

To examine whether the functional connectivity profiles were segregated by BF nuclei, we employed hierarchical clustering using Euclidean distance and Ward's criterion (implemented with scipy; Jones et al. 2001) on voxel-wise rsFC values computed from the whole BF ROI mask (95 voxels). Ward clustering was selected over other clustering mechanisms due to previous reports of its superior accuracy and reproducibility in parcellating functional MRI data (Thirion et al., 2014). We were particularly interested in a $k=2$ cluster solution to directly examine if the two anatomical nuclei were the predominant, discriminable clusters within the functional data; however, solutions were generated for all cluster sizes. The most parsimonious solution was selected by choosing the one with the maximum silhouette score (implemented with scikit-learn; Pedregosa et al. 2011). Silhouette scores provide a metric for how similar elements of a given cluster are to each other compared to elements from other clusters; a higher silhouette score suggests a more appropriate clustering solution (Rousseeuw, 1987). Clusters were independently generated for multi- and single-echo data.

\subsection{Partial correlations}

To ensure that contributions of each BF nucleus to their derived functional connectivity profiles were unique, we conducted partial correlations on each nucleus, linearly regressing out signal from the other. To examine shared connectivity between the nuclei, we used a simple conjunction (boolean "AND") on the results of these analyses (Nichols et al., 2005). Distinct connectivity patterns for each nucleus were examined with a simple disjunction (boolean "NOT") of the shared patterns.

\subsection{Thresholding}

Group-level functional connectivity maps are presented as Z-scores, thresholded at $p \leq 0.001$ uncorrected and cluster-corrected to $p \leq 0.01$. Given the sample size of the current study, using an uncorrected $p \leq 0.001$ threshold ensured all results were also significant at the FDR $q \leq 0.05$ threshold. Group analyses and cluster correction for functional connectivity maps were implemented using AFNI's 3dttest++ command with the -clustim option, which generates approximately 10,000 permuted versions of the group-level residuals to calculate appropriate cluster thresholds based on 
Table 1. Dice coefficients of functional clusters and BF nuclei. Functional clusters were generated via Ward clustering using voxel-wise resting-state functional connectivity maps from the BF with a $k=2$ cluster solution. Functional cluster overlap with BF nuclei was calculated using the Dice coefficient. Labels c1 and c2 correspond respectively to yellow and blue clusters in Figure 3.

\begin{tabular}{lrrrrrrr}
\hline & \multicolumn{2}{c}{ Single-echo } & & \multicolumn{2}{c}{ Multi-echo } \\
\cline { 2 - 3 } \cline { 6 - 8 } & & $\mathrm{c} 1$ & $\mathrm{c} 2$ & & $\mathrm{c} 1$ & $\mathrm{c} 2$ \\
\hline Nucleus basalis of Meynert (NBM) & & 0 & 0.99 & & 0 & 0.91 \\
Medial septum / diagonal band (MS/DB) & & 0.93 & 0 & & 0.96 & 0.06 \\
\hline
\end{tabular}

intrinsic smoothness of the data. Despite recent concerns with this implementation of Type I error correction (Eklund et al., 2016), this method of cluster-correction appropriately attenuates the family-wise error rate to under 0.05 (Cox et al., 2016). All group-level results are presented using FSLeyes software (https://git.fmrib.ox.ac.uk/fsl/fsleyes/fsleyes). Inflated statistical maps can be found in Figure 5; full statistical maps can be viewed online at NeuroVault (https://neurovault.org/collections/QAMPLTIB).

\section{Results}

\subsection{Basal forebrain resting-state functional connectivity}

We assessed the functional connectivity profiles of the BF in healthy adults using multi-echo independent components regression and traditional single-echo time-series functional correlations. Group-level effects were calculated with independent one-sample t-tests.

Functional connectivity of the BF was first examined with ME-ICR, which computes the correlation between BOLD-identified component weight vectors generated via ME-ICA. Results revealed broad relationships with all of insulo-opercular cortex, the cingulum band, medial prefrontal cortex, and bilateral fusiform gyrus (see Figure 2A). Additionally, strong connections were present between the NBM and subcortical targets, including the amygdala and thalamus, extending into the cerebellum. A full list of regions can be found in Table 2 .

Resting state functional connectivity ( $\mathrm{rsFC}$ ) of the BF was also assessed using traditional timeseries correlations with single-echo processed data (Figure 2B). Similar connections were found between the $\mathrm{BF}$ and prefrontal cortex, insulo-opercular cortex, and subcortical features (i.e., the amygdala; Table 2).

\subsection{Functional clustering}

We used Ward clustering to examine the segregation of the BF nuclei based on functional connectivity and the potential correspondence with anatomy. Voxel-wise rsFC maps for both singleand multi-echo data were calculated for every voxel in the whole BF ROI against the rest of the brain and subjected to Ward clustering. Notably, for both single (0.3424) and multi-echo data $(0.2275)$ silhouette scores were highest for a $k=2$ solution; these clustering solutions were carried forward for further analysis (Figure 3). Overlap with anatomical nuclei was calculated using the Sorensen-Dice index (Zou et al., 2004).

Multi-echo data generated two clusters of size 34 (c1) and 61 (c2) voxels, with very high affinity to anatomical delineations of BF nuclei (see Table 1 for Dice coefficients). Single-echo data resulted 
Table 2. Basal forebrain functional connectivity. Regions functionally connected with the BF as assessed via ME-ICR (multi-echo) and time-series rsFC (single-echo). Coordinates are provided in MNI (Montreal Neurological Institute) space. $k$ values represent the voxel extent of the given cluster and $Z$ values are from the peak coordinate in the cluster. Sub-peaks were assessed within larger clusters based on a $|Z|>10$ threshold and a minimum distance $\geq 10 \mathrm{~mm}$ from other sub-peaks; locations for sub-peaks are italicized.

\begin{tabular}{|c|c|c|c|c|c|}
\hline & $x$ & $y$ & $z$ & $k$ & $Z$ \\
\hline \multicolumn{6}{|l|}{ Multi-echo results } \\
\hline Right ventral striatum & 23 & 10 & -5 & 45800 & 12.65 \\
\hline Left putamen & -23 & 10 & -3 & & 12.42 \\
\hline Right caudate nucleus & 13 & 14 & 7 & & 11.41 \\
\hline Right caudate nucleus & 11 & 16 & -5 & & 11.33 \\
\hline Left caudate nucleus & -7 & 14 & -7 & & 10.98 \\
\hline Right putamen & 33 & -4 & -5 & & 10.96 \\
\hline Left putamen & -33 & -6 & -5 & & 10.78 \\
\hline Left amygdala & -23 & 0 & -15 & & 10.17 \\
\hline Left caudate nucleus & -11 & 10 & 7 & & 10.13 \\
\hline Left angular gyrus & -45 & -62 & 29 & 412 & 4.65 \\
\hline Left superior frontal gyrus & -19 & 38 & 45 & 113 & 4.67 \\
\hline Left lingual gyrus & -3 & -86 & -7 & 70 & 4.17 \\
\hline Right precentral gyrus & 25 & -28 & 61 & 58 & 4.29 \\
\hline Left inferior frontal gyrus & -37 & 28 & -1 & 56 & 4.14 \\
\hline Right middle frontal gyrus & 39 & 18 & 25 & 54 & 4.25 \\
\hline Left precentral gyrus & -21 & -26 & 59 & 37 & 4.20 \\
\hline \multicolumn{6}{|l|}{ Single-echo results } \\
\hline Right ventral striatum & 23 & 10 & -5 & 23106 & 12.74 \\
\hline Left putamen & -23 & 8 & -5 & & 12.63 \\
\hline Right caudate nucleus & 11 & 12 & -11 & & 12.23 \\
\hline Left caudate nucleus & -9 & 10 & -11 & & 12.08 \\
\hline Right amygdala & 23 & -4 & -17 & & 12.00 \\
\hline Left caudate nucleus & -9 & 16 & -5 & & 11.91 \\
\hline Right caudate nucleus & 13 & 18 & -1 & & 11.77 \\
\hline Left amygdala & -21 & -4 & -17 & & 11.74 \\
\hline Right thalamus & 1 & -12 & 5 & & 11.13 \\
\hline Left hippocampus & -27 & -14 & -19 & & 10.90 \\
\hline Left anterior cingulate cortex & -5 & 46 & 7 & & 10.46 \\
\hline Left anterior cingulate cortex & -5 & 46 & -1 & & 10.40 \\
\hline Hypothalamus & 7 & 2 & -11 & & 10.26 \\
\hline
\end{tabular}


A
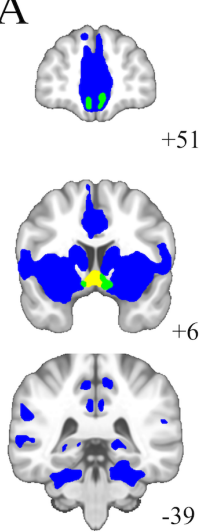

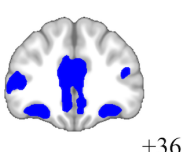

$+36$
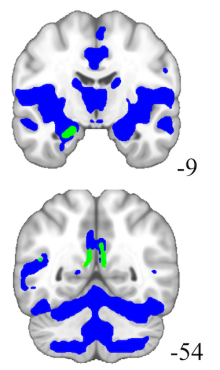
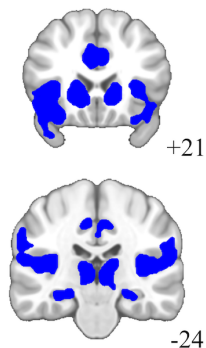

$-24$

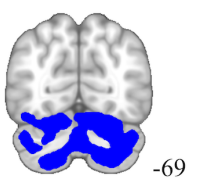

B
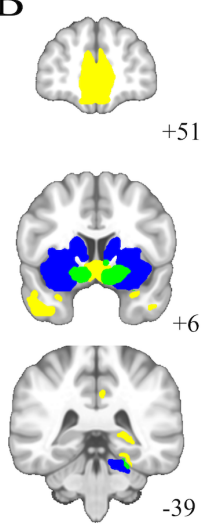
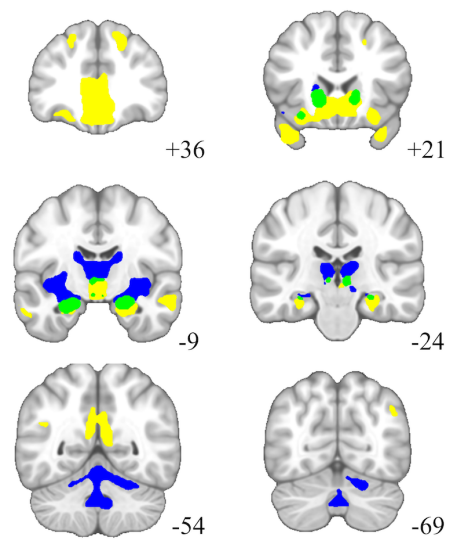

Figure 4. Functional correlations of the medial septum and diagonal band (MS/DB, Ch123) and nucleus basalis of Meynert (NBM, Ch4) for A. multi-echo preprocessed and B. single-echo preprocessed data. Yellow denotes connectivity specific to the MS/DB, blue specific to the NBM, and green is shared connectivity.

in clusters of size 43 (c1) and 52 (c2) voxels, respectively (Table 1).

An additional clustering analysis was done focusing solely on intra-BF connectivity: rsFC maps from within the $\mathrm{BF}$ were used as the input to a $k=2$ clustering. Results were similar to the whole-brain analysis: multi-echo data yielded two clusters of size $49(\mathrm{NBM}=0.94, \mathrm{MS} / \mathrm{DB}=0)$ and $46(\mathrm{NBM}=0.08, \mathrm{MS} / \mathrm{DB}=0.89)$ voxels respectively, while single-echo yielded clusters of size $46(\mathrm{NBM}=0.93, \mathrm{MS} / \mathrm{DB}=0)$ and $49(\mathrm{NBM}=0.12, \mathrm{MS} / \mathrm{DB}=0.86)$ voxels. The clusters followed anatomical delineations moderately well, but less so than those generated via whole-brain voxel-wise clustering.

\subsection{Basal forebrain nuclei rsFC}

Given the alignment of functional clustering of the BF with anatomical divisions, we independently examined potentially distinct functional profiles of the BF nuclei: the NBM and the MS/DB. As the functional parcels derived via Ward clustering demonstrated high correspondence with the anatomical ROIs (see Table 1), we opted to use the latter in order to examine the individual profiles of the subnuclei. Importantly, using the anatomical ROIs allowed us to continue to investigate the similarities between the single- and multi-echo analyses without biasing results.

In order to better assess shared and distinct functional correlations between the NBM and $\mathrm{MS} / \mathrm{DB}$, partial correlations were conducted on each nucleus, regressing out signal from the other, to ensure contributions were unique to the nucleus of interest.

\subsubsection{Shared NBM and MS/DB connectivity}

A simple conjunction between the NBM and MS/DB functional correlation maps revealed regions with shared connectivity between the two BF nuclei (Figure 4; green regions). In multi-echo data, these connections are primarily found in the hippocampus, posterior cingulate cortex, and ventromedial prefrontal cortex (vmPFC), whereas overlap is localized to the hippocampus and caudate in single-echo data. 
Table 3. Nucleus basalis of Meynert (NBM) functional connectivity. Regions functionally connected with the NBM as assessed via ME-ICR (multi-echo) and time-series rsFC (single-echo). Coordinates are provided in MNI space. Sub-peaks were assessed within larger clusters based on a $|Z|>10$ threshold and a minimum distance $\geq 10 \mathrm{~mm}$ from other sub-peaks; locations for sub-peaks are italicized.

\begin{tabular}{lrrrrr}
\hline & $x$ & $y$ & $z$ & $k$ & $Z$ \\
Multi-echo results & & & & & \\
\hline Right ventral striatum & 23 & 10 & -5 & 32704 & 12.69 \\
Left putamen & -23 & -8 & -3 & & 12.57 \\
Right caudate nucleus & 13 & 16 & 3 & & 11.63 \\
Left caudate nucleus & -11 & -12 & 5 & & 11.39 \\
Right putamen & 33 & -4 & -5 & & 11.09 \\
Right thalamus & 7 & -14 & 9 & & 10.64 \\
Right insula & 41 & 10 & -5 & & 10.60 \\
Left thalamus & -7 & -16 & 9 & & 10.15 \\
Left amygdala & -23 & 0 & -15 & & 10.07 \\
Left insula & -39 & 6 & -7 & & 10.03 \\
Left anterior cingulate cortex & -3 & 42 & 11 & 7947 & 8.24 \\
Left middle temporal gyrus & -57 & -8 & -13 & 151 & 4.38 \\
Right middle frontal gyrus & 41 & 16 & 27 & 151 & 4.54 \\
Left middle temporal gyrus & -55 & -62 & 7 & 101 & 4.29 \\
Left middle frontal gyrus & -39 & 32 & 17 & 75 & 4.34 \\
Single-echo results & & & & & \\
\hline Right ventral striatum & & & & & \\
Left putamen & 23 & 10 & -5 & 9080 & 12.63 \\
Right amygdala & -23 & 8 & -5 & & 12.42 \\
Left amygdala & 25 & -2 & -17 & & 11.40 \\
Right caudate nucleus & -21 & -4 & -15 & & 11.27 \\
Right thalamus & 15 & 4 & 15 & & 10.57 \\
Left thalamus & 5 & -12 & 9 & & 10.19 \\
Right cerebellum (nodule) & -3 & -14 & 7 & & 10.11 \\
\hline & 1 & -58 & -33 & 1888 & 7.41 \\
\hline
\end{tabular}


Table 4. Medial septum/diagonal band of Broca (MS/DB) functional connectivity. Regions functionally connected with the MS/DB as assessed via ME-ICR (multi-echo) and time-series rsFC (single-echo). Coordinates are provided in MNI space. Sub-peaks were assessed within larger clusters based on a $|Z|>10$ threshold and a minimum distance $\geq 5 \mathrm{~mm}$ from other sub-peaks; locations for sub-peaks are italicized.

\begin{tabular}{lrrrrr}
\hline & $x$ & $y$ & $z$ & $k$ & $Z$ \\
Multi-echo results & & & & & \\
\hline Medial septal nucleus & -5 & 2 & -11 & 635 & 7.25 \\
Right medial frontal gyrus & 5 & 48 & -17 & 229 & 4.29 \\
Right superior temporal gyrus & 49 & -58 & 19 & 144 & 4.27 \\
Right parahippocampal gyrus & 27 & -16 & -19 & 121 & 4.18 \\
Left posterior cingulate cortex & -5 & -52 & 25 & 106 & 3.87 \\
Left anterior cingulate cortex & -5 & 46 & 1 & 85 & 4.04 \\
Right posterior cingulate cortex & 5 & -56 & 21 & 83 & 3.94 \\
& & & & & \\
Single-echo results & & & & & \\
\hline Medial septal nucleus & 11 & 12 & -11 & 10564 & 12.20 \\
Left caudate nucleus & -7 & 12 & -9 & & 12.06 \\
Left anterior cingulate cortex & -5 & 46 & -1 & & 10.38 \\
Left hippocampus & -25 & -14 & -19 & & 10.37 \\
Hypothalamus & 7 & 2 & -11 & & 10.21 \\
Left posterior cingulate & -7 & -52 & 17 & 784 & 4.99 \\
Left superior frontal gyrus & -19 & 40 & 43 & 263 & 5.64 \\
Left middle temporal gyrus & -61 & -10 & -17 & 232 & 4.42 \\
Right superior temporal gyrus & 51 & -60 & 27 & 189 & 4.66 \\
Right middle frontal gyrus & 21 & 32 & 47 & 156 & 4.73 \\
Left angular gyrus & -47 & -64 & 33 & 140 & 4.66 \\
\hline
\end{tabular}




\subsubsection{Distinct NBM and MS/DB connectivity}

Examining functional connectivity of the $\mathrm{Ch} 4$ group of the BF (NBM) using ME-ICR revealed a similar profile to that of the entire BF ROI (Figure 4A, blue), with relationships to prefrontal cortex, the cingulum band, insulo-opercular cortex, fusiform gyrus, and subcortical features like the amygdala. Two additional clusters in the left inferior frontal gyrus and left postcentral gyrus were observed that had not been found in the whole BF analysis (Table 3).

Compared to ME-ICR, single-echo analyses showed more circumscribed connectivity for the NBM (Figure 4B, blue). Connections were noted with insulo-opercular cortex and subcortical targets, extending into the cerebellum (Table 3).

In contrast to the NBM, functional correlations from the Ch1-3 group of the BF (MS/DB) were more localized (Figure 4A, yellow). Multi-echo processed data revealed distinct connections between the MS/DB and the caudate (Table 4).

Single-echo processed data revealed broader connections from the MS/DB than were observed in multi-echo data (Figure 4B, yellow). Significant correlations included prefrontal cortex, medial temporal lobes, and posterior cingulate, with additional connections to the hippocampus and orbitofrontal cortex (Table 4).

\section{Discussion}

We assessed whole-brain, resting-state functional connectivity (rsFC) of the basal forebrain (BF) with multi- and single-echo fMRI data. The results demonstrate that the human BF supports broad functional connections with neocortical and subcortical regions, consistent with its prominent neuromodulatory role in nonhuman animals. Voxel-wise functional connectivity data from the BF were subjected to Ward clustering to examine functionally-defined BF nuclei, revealing almost perfect alignment between functional data and anatomical nuclei. Further rsFC analyses were conducted to examine the potential differential functional profiles of these anatomical nuclei. The nucleus basalis of Meynert (NBM) shows widespread functional correlations throughout lateral neocortex and subcortical regions, including the thalamus and amygdala, while connections with the medial septum/diagonal band of Broca (MS/DB) are more functionally connected to structures associated with the hippocampal formation.

\subsection{Basal forebrain nuclei functional networks}

The present results support a functional segregation of BF nuclei in the human brain. Clustering of human BF connections yielded outputs with high correspondence to anatomical delineations found in nonhuman animals. Moreover, the functional profiles were distinct for the NBM and MS/DB. Our results are in line with prior studies demonstrating a correspondence between anatomical connections and $\mathrm{rsFC}$ in humans and nonhuman primates (Genç et al., 2015; Hagmann et al., 2008; Honey et al., 2009; Margulies et al., 2009; Miranda-Dominguez et al., 2014; Mišić et al., 2016; Skudlarski et al., 2008).

Although the basal forebrain has a complex neurochemical profile (Lin et al., 2015), the functional connections from the MS/DB appeared to follow well-established cholinergic relationships, with observed correlations to the hippocampus, orbitofrontal cortex, and parahippocampal gyrus (Hedreen et al., 1984; Mesulam et al., 1983a,b). Although not directly assessed in the current study, the specificity of the functional profile revealed with these rs-fMRI data suggests that we may be observing relationships made through MS/DB cholinergic projections, rather than through the diffuse MS/DB GABAergic/glutamatergic network of projections (Mesulam et al., 1983b).

As hypothesized, we observed broad temporal correlations from the NBM to the amygdala and such neocortical regions as the insulo-opercular cortex, cingulum band, and prefrontal cortex. Ho- 
bioRxiv preprint doi: https://doi.org/10.1101/211086; this version posted October 30, 2017. The copyright holder for this preprint (which was not certified by peer review) is the author/funder, who has granted bioRxiv a license to display the preprint in perpetuity. It is made available under aCC-BY 4.0 International license.

\section{Multi-echo}

A
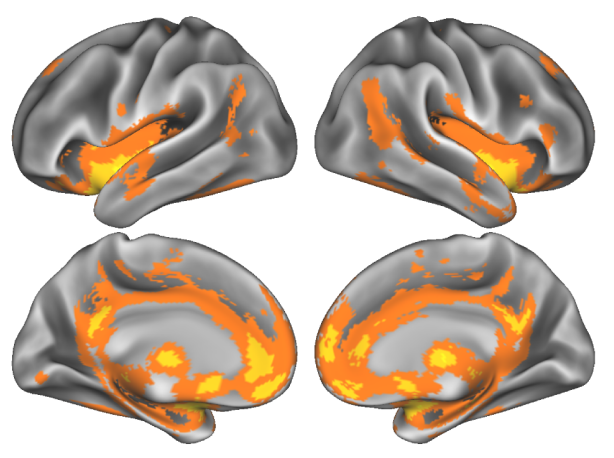

$\mathrm{B}$
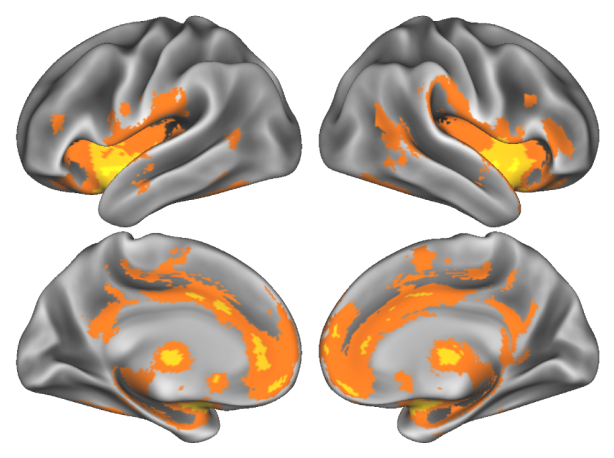

$\mathrm{C}$
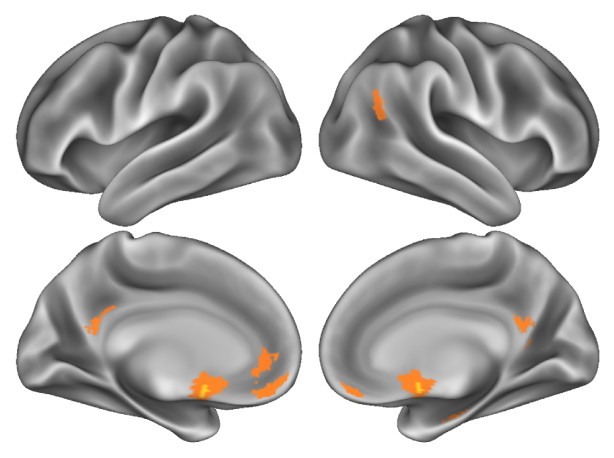

\section{Single-echo}
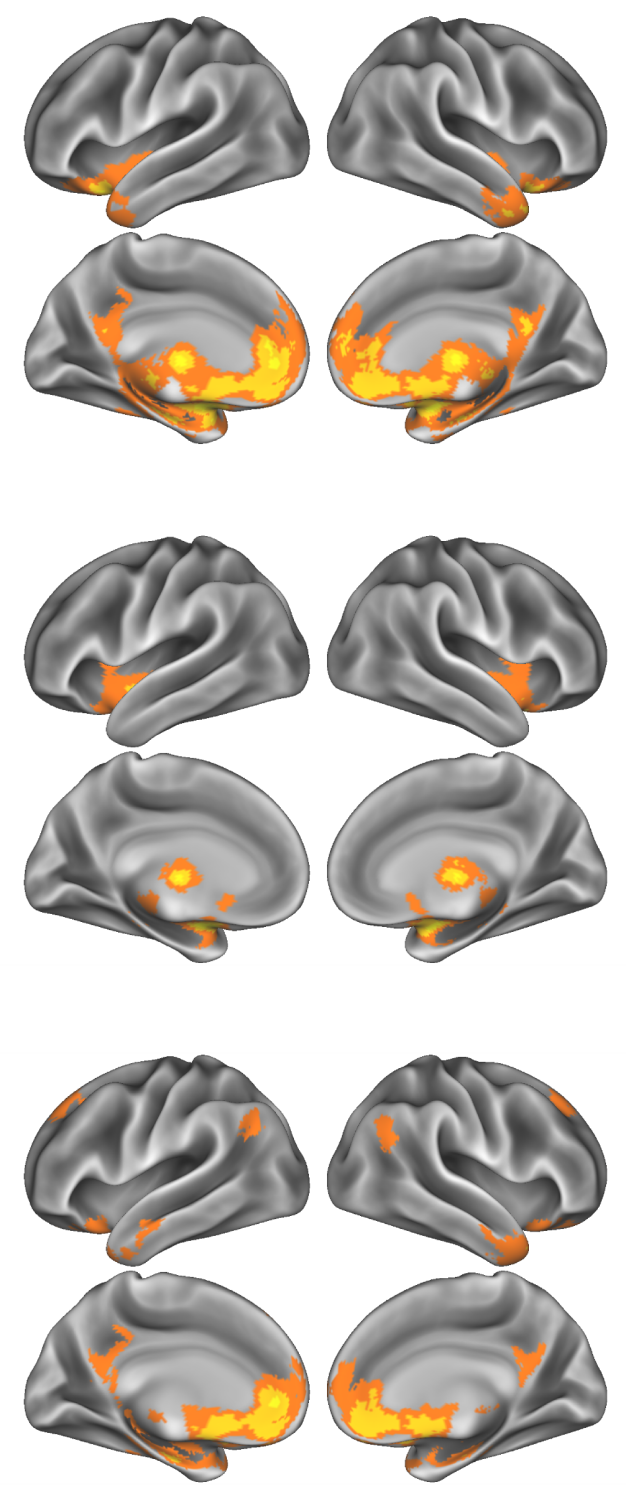

Figure 5. Inflated (i.e., surface) projections of multi- and single-echo functional connectivity results. A. Basal forebrain, B. NBM, and C. MS/DB ROI functional connectivity. Figure was generated using the Connectome Workbench software (Marcus et al., 2011). 
mologous brain regions in rodent and nonhuman primate tracing studies have demonstrated similar strong cholinergic innervations from the NBM (Mesulam et al., 1983a, 1986, 1983b; Zaborszky et al., 2015a,b). Notable in their absence were functional connections with extrastriate cortical areas, which are also known to have anatomical connections with the NBM; however, this could be due to the nature of resting-state acquisition, for which no external stimuli were present, as this functional relationship has been observed in a previous human fMRI study during an experimental task with color stimuli (De Rosa et al., 2004).

\subsection{Resting-state networks and the basal forebrain}

Both the NBM and MS/DB showed distinct functional connectivity with regions typically associated with the default network (DN), including ventromedial prefrontal cortex (vmPFC), posterior cingulate cortex (PCC), angular gyrus, and the medial temporal lobes (Andrews-Hanna et al., 2014). While these regions are known to be innervated by BF projections, it appears BF targets do not fit neatly into the canonical human resting-state functional networks. Indeed, NBM rsFC analyses showed connectivity with regions that included the Default, Frontoparietal Control (FPCN; dorsolateral prefrontal cortex), and Ventral Attention (VAN; insula) networks (Yeo et al., 2011). This is consistent with the BF as the source of a broad neuromodulatory network, playing a critical role across different facets of cognition and orchestrating information across these distinct resting-state networks.

\subsection{Single- and multi-echo analysis}

Despite previous reports of superior specificity of results and validity of statistical inferences with multi-echo fMRI data (Kundu et al., 2013, 2017), the current study suggests a more nuanced distinction between single- and multi-echo results. We compared multi-echo independent components analysis with a rigorous single-echo preprocessing pipeline (Power et al., 2012, 2014). Both the multi- and single-echo analysis pipelines detected discriminable functional networks from the BF nuclei (Figure 3); while multi-echo data seemed to provide greater detection power for the NBM functional network (Figure 4), the opposite appeared to be true for the MS/DB network (Figure 4).

It is worthwhile to note that the multi-echo preprocessing pipeline, which is entirely data-driven, retains many of the features of the original signal that are removed in the single-echo pipeline (i.e., by bandpass filtering, Gaussian smoothing) while still adequately attenuating noise (Figure 1). In this respect, multi-echo results may provide a truer snapshot of the underlying neural dynamics and connectivity profiles. We recommend future studies utilizing multi-echo data conduct joint analyses with single- and multi-echo processing pipelines to continue to provide a better picture of their unique benefits.

\subsection{Methodological considerations}

Our BOLD data, although likely reflecting underlying neural activity, are limited in lack of neurotransmitter specificity. Nonetheless, the observed connectivity patterns are consistent with known cholinergic projections of the BF (Gritti et al., 1994, 1997, 1998; Jourdain et al., 1989; Mesulam et al., 1983b; Meynert, 1872; Parent et al., 1988; Unal et al., 2015). Future studies could make direct assessments of cholinergic contributions by comparing network connectivity changes with systemic cholinergic pharmacology in humans.

\subsection{Conclusions}

Our current findings demonstrate differential functional correlation profiles of human BF nuclei. The NBM had diffuse connections with neocortical regions and the amygdala, while the MS/DB showed circumscribed relationships with the hippocampus and parahippocampal complex. These 
connections appear to support homologous anatomical relationships to the nonhuman rodent and primate literature (Mesulam et al., 1983a, 1986, 1983b). Given the importance of the BF as a key neuromodulatory hub, understanding these relationships is an important first step towards better characterizing the role of the BF in neural dynamics, its support of cognition, and its decline with neurodegeneration in aging.

\section{Acknowledgments}

The authors wish to thank Elizabeth DuPre for helpful feedback on an original version of this manuscript. This work was supported by an Empire Innovation Program Grant [88021813] to E.D.R and an NIH NCRR grant [1S10RR025145] to the Cornell MRI Facility.

\section{Conflicts of interest}

None.

\section{References}

Andrews-Hanna, J. R., Smallwood, J., and Spreng, R. N. (2014). The default network and self-generated thought: component processes, dynamic control, and clinical relevance. Annals of the New York Academy of Sciences, 1316(1):29-52.

Baxter, M. G. and Bucci, D. J. (2013). Selective immunotoxic lesions of basal forebrain cholinergic neurons: twenty years of research and new directions. Behavioral Neuroscience, 127(5):611-618.

Baxter, M. G., Bucci, D. J., Gorman, L. K., Wiley, R. G., and Gallagher, M. (1995). Selective immunotoxic lesions of basal forebrain cholinergic cells: effects on learning and memory in rats. Behavioral Neuroscience, 109(4):714.

Bell, P. T. and Shine, J. M. (2016). Subcortical contributions to large-scale network communication. Neuroscience $\mathscr{E}$ Biobehavioral Reviews, 71:313-322.

Botly, L. C. and De Rosa, E. (2007). Cholinergic influences on feature binding. Behavioral Neuroscience, 121(2):264.

Botly, L. C. and De Rosa, E. (2008). A cross-species investigation of acetylcholine, attention, and feature binding. Psychological Science, 19(11):1185-1193.

Botly, L. C. and De Rosa, E. (2009). Cholinergic deafferentation of the neocortex using 192 IgG-saporin impairs feature binding in rats. Journal of Neuroscience, 29(13):4120-4130.

Botly, L. C. and De Rosa, E. (2012). Impaired visual search in rats reveals cholinergic contributions to feature binding in visuospatial attention. Cerebral Cortex, 22(10):2441-2453.

Brashear, H. R., Zaborszky, L., and Heimer, L. (1986). Distribution of GABAergic and cholinergic neurons in the rat diagonal band. Neuroscience, 17(2):439-451.

Chudasama, Y., Dalley, J. W., Nathwani, F., Bouger, P., and Robbins, T. W. (2004). Cholinergic modulation of visual attention and working memory: dissociable effects of basal forebrain 192-IgG-saporin lesions and intraprefrontal infusions of scopolamine. Learning \& Memory, 11(1):78-86.

Collins, D. L., Neelin, P., Peters, T. M., and Evans, A. C. (1994). Automatic 3D intersubject registration of MR volumetric data in standardized Talairach space. Journal of Computer Assisted Tomography, 18(2):192-205.

Cox, R. W. (1996). AFNI: software for analysis and visualization of functional magnetic resonance neuroimages. Computers and Biomedical Research, 29(3):162-173. 
Cox, R. W., Reynolds, R. C., and Taylor, P. A. (2016). AFNI and Clustering: False Positive Rates Redux. bioRxiv.

Coyle, J. T., Price, D. L., and Delong, M. R. (1983). Alzheimer's disease: a disorder of cortical cholinergic innervation. Science, 219(4589):1184-1190.

Croxson, P. L., Kyriazis, D. A., and Baxter, M. G. (2011). Cholinergic modulation of a specific memory function of prefrontal cortex. Nature Neuroscience, 14(12):1510-1512.

Davies, P. and Maloney, A. (1976). Selective loss of central cholinergic neurons in Alzheimer's disease. The Lancet, 308(8000):1403.

De Rosa, E., Desmond, J. E., Anderson, A. K., Pfefferbaum, A., and Sullivan, E. V. (2004). The human basal forebrain integrates the old and the new. Neuron, 41(5):825-837.

Descarries, L., Mechawar, N., Aznavour, N., and Watkins, K. C. (2004). Structural determinants of the roles of acetylcholine in cerebral cortex. Progress in Brain Research, 145:45-58.

Dipasquale, O., Sethi, A., Laganà, M. M., Baglio, F., Baselli, G., Kundu, P., Harrison, N. A., and Cercignani, M. (2017). Comparing resting state fMRI de-noising approaches using multi-and single-echo acquisitions. PloS one, 12(3):e0173289.

DuPre, E., Luh, W.-M., and Spreng, R. N. (2016). Multi-echo fMRI replication sample of autobiographical memory, prospection and theory of mind reasoning tasks. Scientific Data, 3.

Eickhoff, S. B., Paus, T., Caspers, S., Grosbras, M. H., Evans, A. C., Zilles, K., and Amunts, K. (2007). Assignment of functional activations to probabilistic cytoarchitectonic areas revisited. NeuroImage, $36(3): 511-521$.

Eklund, A., Nichols, T. E., and Knutsson, H. (2016). Cluster failure: Why fMRI inferences for spatial extent have inflated false-positive rates. Proceedings of the National Academy of Sciences, 113(28):7900-7905.

Emre, M., Heckers, S., Mash, D. C., Geula, C., Mesulam, M., et al. (1993). Cholinergic innervation of the amygdaloid complex in the human brain and its alterations in old age and alzheimer's disease. Journal of Comparative Neurology, 336(1):117-134.

Genç, E., Schölvinck, M. L., Bergmann, J., Singer, W., and Kohler, A. (2015). Functional connectivity patterns of visual cortex reflect its anatomical organization. Cerebral Cortex, pages 3719-3731.

Golden, S. A., Heshmati, M., Flanigan, M., Christoffel, D. J., Guise, K., Pfau, M. L., Aleyasin, H., Menard, C., Zhang, H., Hodes, G. E., et al. (2016). Basal forebrain projections to the lateral habenula modulate aggression reward. Nature, 534(7609):688-692.

Gorgolewski, K., Burns, C. D., Madison, C., Clark, D., Halchenko, Y. O., Waskom, M. L., and Ghosh, S. S. (2011). Nipype: a flexible, lightweight and extensible neuroimaging data processing framework in python. Frontiers in Neuroinformatics, 5.

Gritti, I., Henny, P., Galloni, F., Mainville, L., Mariotti, M., and Jones, B. E. (2006). Stereological estimates of the basal forebrain cell population in the rat, including neurons containing choline acetyltransferase, glutamic acid decarboxylase or phosphate-activated glutaminase and colocalizing vesicular glutamate transporters. Neuroscience, 143(4):1051-1064.

Gritti, I., Mainville, L., and Jones, B. E. (1993). Codistribution of GABA-with acetylcholine-synthesizing neurons in the basal forebrain of the rat. Journal of Comparative Neurology, 329(4):438-457.

Gritti, I., Mainville, L., and Jones, B. E. (1994). Projections of GABAergic and cholinergic basal forebrain and GABAergic preoptic-anterior hypothalamic neurons to the posterior lateral hypothalamus of the rat. Journal of Comparative Neurology, 339(2):251-268.

Gritti, I., Mainville, L., Mancia, M., and Jones, B. E. (1997). GABAergic and other noncholinergic basal forebrain neurons, together with cholinergic neurons, project to the mesocortex and isocortex in the rat. The Journal of comparative neurology, 383(2):163-177. 
Gritti, I., Mariotti, M., and Mancia, M. (1998). GABAergic and cholinergic basal forebrain and preopticanterior hypothalamic projections to the mediodorsal nucleus of the thalamus in the cat. Neuroscience, $85(1): 149-178$.

Gritton, H. J., Howe, W. M., Mallory, C. S., Hetrick, V. L., Berke, J. D., and Sarter, M. (2016). Cortical cholinergic signaling controls the detection of cues. Proceedings of the National Academy of Sciences, 113(8):E1089-E1097.

Grothe, M., Heinsen, H., and Teipel, S. (2013). Longitudinal measures of cholinergic forebrain atrophy in the transition from healthy aging to Alzheimer's disease. Neurobiology of Aging, 34(4):1210-1220.

Hagmann, P., Cammoun, L., Gigandet, X., Meuli, R., Honey, C. J., Wedeen, V. J., and Sporns, O. (2008). Mapping the structural core of human cerebral cortex. PLoS Biology, 6(7):e159.

Hasselmo, M. E. and Sarter, M. (2011). Modes and models of forebrain cholinergic neuromodulation of cognition. Neuropsychopharmacology, 36(1):52-73.

Hedreen, J. C., Struble, R. G., Whitehouse, P. J., and Price, D. L. (1984). Topography of the magnocellular basal forebrain system in human brain. Journal of Neuropathology \&3 Experimental Neurology, 43(1):1-21.

Holmes, C. J., Hoge, R., Collins, L., Woods, R., Toga, A. W., and Evans, A. C. (1998). Enhancement of MR images using registration for signal averaging. Journal of Computer Assisted Tomography, 22(2):324-333.

Honey, C., Sporns, O., Cammoun, L., Gigandet, X., Thiran, J.-P., Meuli, R., and Hagmann, P. (2009). Predicting human resting-state functional connectivity from structural connectivity. Proceedings of the National Academy of Sciences, 106(6):2035-2040.

Jenkinson, M., Pechaud, M., and Smith, S. (2005). Bet2: Mr-based estimation of brain, skull and scalp surfaces. In Eleventh Annual Meeting of the Organization for Human Brain Mapping, volume 17, page 167. Toronto.

Jones, E., Oliphant, T., Peterson, P., et al. (2001). SciPy: Open source scientific tools for Python. v0.19.0.

Jourdain, A., Semba, K., and Fibiger, H. C. (1989). Basal forebrain and mesopontine tegmental projections to the reticular thalamic nucleus: an axonal collateralization and immunohistochemical study in the rat. Brain research, 505(1):55-65.

Kim, J.-H., Jung, A.-H., Jeong, D., Choi, I., Kim, K., Shin, S., Kim, S. J., and Lee, S.-H. (2016). Selectivity of neuromodulatory projections from the basal forebrain and locus ceruleus to primary sensory cortices. Journal of Neuroscience, 36(19):5314-5327.

Kim, T., Thankachan, S., McKenna, J. T., McNally, J. M., Yang, C., Choi, J. H., Chen, L., Kocsis, B., Deisseroth, K., Strecker, R. E., et al. (2015). Cortically projecting basal forebrain parvalbumin neurons regulate cortical gamma band oscillations. Proceedings of the National Academy of Sciences, 112(11):35353540 .

Kondo, H. and Zaborszky, L. (2016). Topographic organization of the basal forebrain projections to the perirhinal, postrhinal, and entorhinal cortex in rats. Journal of Comparative Neurology, 524(12):25032515 .

Kundu, P., Benson, B. E., Baldwin, K. L., Rosen, D., Luh, W.-M., Bandettini, P. A., Pine, D. S., and Ernst, M. (2015). Robust resting state fMRI processing for studies on typical brain development based on multi-echo EPI acquisition. Brain Imaging and Behavior, 9(1):56-73.

Kundu, P., Brenowitz, N. D., Voon, V., Worbe, Y., Vertes, P. E., Inati, S. J., Saad, Z. S., Bandettini, P. A., and Bullmore, E. T. (2013). Integrated strategy for improving functional connectivity mapping using multiecho fMRI. Proceedings of the National Academy of Sciences, 110(40):16187-16192.

Kundu, P., Inati, S. J., Evans, J. W., Luh, W. M., and Bandettini, P. A. (2012). Differentiating BOLD and non-BOLD signals in fMRI time series using multi-echo EPI. NeuroImage, 60(3):1759-1770.

Kundu, P., Voon, V., Balchandani, P., Lombardo, M. V., Poser, B. A., and Bandettini, P. (2017). Multi-Echo fMRI: A review of applications in fMRI denoising and analysis of BOLD signals. NeuroImage, advance online publication. 
Li, C. S., Ide, J. S., Zhang, S., Hu, S., Chao, H. H., and Zaborszky, L. (2014). Resting state functional connectivity of the basal nucleus of Meynert in humans: in comparison to the ventral striatum and the effects of age. NeuroImage, 97:321-332.

Lin, S.-C., Brown, R. E., Shuler, M. G. H., Petersen, C. C., and Kepecs, A. (2015). Optogenetic dissection of the basal forebrain neuromodulatory control of cortical activation, plasticity, and cognition. Journal of Neuroscience, 35(41):13896-13903.

Ljubojevic, V., Luu, P., and De Rosa, E. (2014). Cholinergic contributions to supramodal attentional processes in rats. Journal of Neuroscience, 34(6):2264-2275.

Lysakowski, A., Wainer, B., Bruce, G., and Hersh, L. (1989). An atlas of the regional and laminar distribution of choline acetyltransferase immunoreactivity in rat cerebral cortex. Neuroscience, 28(2):291-336.

Ma, M. and Luo, M. (2012). Optogenetic activation of basal forebrain cholinergic neurons modulates neuronal excitability and sensory responses in the main olfactory bulb. Journal of Neuroscience, 32(30):1010510116.

Manns, I. D., Mainville, L., and Jones, B. E. (2001). Evidence for glutamate, in addition to acetylcholine and GABA, neurotransmitter synthesis in basal forebrain neurons projecting to the entorhinal cortex. Neuroscience, 107(2):249-263.

Marcus, D., Harwell, J., Olsen, T., Hodge, M., Glasser, M., Prior, F., Jenkinson, M., Laumann, T., Curtiss, S., and Van Essen, D. (2011). Informatics and data mining tools and strategies for the Human Connectome Project. Frontiers in Neuroinformatics, 5:4.

Margulies, D. S., Vincent, J. L., Kelly, C., Lohmann, G., Uddin, L. Q., Biswal, B. B., Villringer, A., Castellanos, F. X., Milham, M. P., and Petrides, M. (2009). Precuneus shares intrinsic functional architecture in humans and monkeys. Proceedings of the National Academy of Sciences, 106(47):20069-20074.

Mesulam, M., Geula, C., et al. (1988). Nucleus basalis (ch4) and cortical cholinergic innervation in the human brain: observations based on the distribution of acetylcholinesterase and choline acetyltransferase. Journal of Comparative Neurology, 275(2):216-240.

Mesulam, M., Mufson, E., Wainer, B., and Levey, A. (1983a). Central cholinergic pathways in the rat: an overview based on an alternative nomenclature (Ch1-Ch6). Neuroscience, 10(4):1185-1201.

Mesulam, M., Volicer, L., Marquis, J. K., Mufson, E. J., Green, R. C., et al. (1986). Systematic regional differences in the cholinergic innervation of the primate cerebral cortex: distribution of enzyme activities and some behavioral implications. Annals of Neurology, 19(2):144-151.

Mesulam, M. M., Mufson, E. J., Levey, A. I., and Wainer, B. H. (1983b). Cholinergic innervation of cortex by the basal forebrain: cytochemistry and cortical connections of the septal area, diagonal band nuclei, nucleus basalis (substantia innominata), and hypothalamus in the rhesus monkey. Journal of Comparative Neurology, 214(2):170-197.

Meynert, T. (1872). The brain of mammals. A manual of histology, pages 650-766.

Miranda-Dominguez, O., Mills, B. D., Grayson, D., Woodall, A., Grant, K. A., Kroenke, C. D., and Fair, D. A. (2014). Bridging the gap between the human and macaque connectome: a quantitative comparison of global interspecies structure-function relationships and network topology. Journal of Neuroscience, $34(16): 5552-5563$.

Mišić, B., Betzel, R. F., De Reus, M. A., Van Den Heuvel, M. P., Berman, M. G., McIntosh, A. R., and Sporns, O. (2016). Network-level structure-function relationships in human neocortex. Cerebral Cortex, 26(7):3285-3296.

Muir, J. L. (1997). Acetylcholine, aging, and Alzheimer's disease. Pharmacology, Biochemistry and Behavior, 56(4):687-696.

Muñoz, W. and Rudy, B. (2014). Spatiotemporal specificity in cholinergic control of neocortical function. Current Opinion in Neurobiology, 26:149-160. 
Murphy, K., Birn, R. M., Handwerker, D. A., Jones, T. B., and Bandettini, P. A. (2009). The impact of global signal regression on resting state correlations: are anti-correlated networks introduced? Neuroimage, 44(3):893-905.

Nichols, T., Brett, M., Andersson, J., Wager, T., and Poline, J.-B. (2005). Valid conjunction inference with the minimum statistic. Neuroimage, 25(3):653-660.

Ovsepian, S. V., O'Leary, V. B., and Zaborszky, L. (2016). Cholinergic mechanisms in the cerebral cortex beyond synaptic transmission. The Neuroscientist, 22(3):238-251.

Parent, A., Pare, D., Smith, Y., and Steriade, M. (1988). Basal forebrain cholinergic and noncholinergic projections to the thalamus and brainstem in cats and monkeys. Journal of Comparative Neurology, 277(2):281-301.

Parikh, V., Kozak, R., Martinez, V., and Sarter, M. (2007). Prefrontal acetylcholine release controls cue detection on multiple timescales. Neuron, 56(1):141-154.

Pedregosa, F., Varoquaux, G., Gramfort, A., Michel, V., Thirion, B., Grisel, O., Blondel, M., Prettenhofer, P., Weiss, R., Dubourg, V., Vanderplas, J., Passos, A., Cournapeau, D., Brucher, M., Perrot, M., and Duchesnay, E. (2011). Scikit-learn: Machine learning in Python. Journal of Machine Learning Research, 12:2825-2830.

Pepeu, G. and Giovannini, M. G. (2017). The fate of the brain cholinergic neurons in neurodegenerative diseases. Brain Research, 1670:173-184.

Perry, E., Perry, R., Blessed, G., and Tomlinson, B. (1977). Necropsy evidence of central cholinergic deficits in senile dementia. The Lancet, 309(8004):189.

Picciotto, M. R., Higley, M. J., and Mineur, Y. S. (2012). Acetylcholine as a neuromodulator: cholinergic signaling shapes nervous system function and behavior. Neuron, 76(1):116-129.

Power, J. D., Barnes, K. A., Snyder, A. Z., Schlaggar, B. L., and Petersen, S. E. (2012). Spurious but systematic correlations in functional connectivity mri networks arise from subject motion. NeuroImage, 59(3):2142-2154.

Power, J. D., Mitra, A., Laumann, T. O., Snyder, A. Z., Schlaggar, B. L., and Petersen, S. E. (2014). Methods to detect, characterize, and remove motion artifact in resting state fmri. NeuroImage, 84:320-341.

Rasmusson, D. (2000). The role of acetylcholine in cortical synaptic plasticity. Behavioural Brain Research, $115(2): 205-218$.

Rousseeuw, P. J. (1987). Silhouettes: a graphical aid to the interpretation and validation of cluster analysis. Journal of computational and applied mathematics, 20:53-65.

Rye, D. B., Wainer, B. H., Mesulam, M. M., Mufson, E. J., and Saper, C. B. (1984). Cortical projections arising from the basal forebrain: a study of cholinergic and noncholinergic components employing combined retrograde tracing and immunohistochemical localization of choline acetyltransferase. Neuroscience, 13(3):627-643.

Saad, Z. S., Gotts, S. J., Murphy, K., Chen, G., Jo, H. J., Martin, A., and Cox, R. W. (2012). Trouble at rest: how correlation patterns and group differences become distorted after global signal regression. Brain connectivity, 2(1):25-32.

Saper, C. and Chelimsky, T. (1984). A cytoarchitectonic and histochemical study of nucleus basalis and associated cell groups in the normal human brain. Neuroscience, 13(4):1023-1037.

Sarter, M. and Bruno, J. P. (1997). Cognitive functions of cortical acetylcholine: toward a unifying hypothesis. Brain Research Reviews, 23(1):28-46.

Saunders, A., Granger, A. J., and Sabatini, B. L. (2015). Corelease of acetylcholine and GABA from cholinergic forebrain neurons. eLife, 4:e06412.

Schmitz, T. W., Spreng, R. N., and Initiative, T. A. D. N. (2016). Basal forebrain degeneration precedes and predicts the cortical spread of Alzheimer's pathology. Nature Communications, 7. 
Selden, N. R., Gitelman, D. R., Salamon-Murayama, N., Parrish, T. B., and Mesulam, M.-M. (1998). Trajectories of cholinergic pathways within the cerebral hemispheres of the human brain. Brain: a journal of neurology, 121(12):2249-2257.

Shine, J., van den Brink, R., Hernaus, D., Nieuwenhuis, S., and Poldrack, R. (2017). Catecholaminergic manipulation alters dynamic network topology across behavioral states. bioRxiv, page 169102.

Shinoe, T., Matsui, M., Taketo, M. M., and Manabe, T. (2005). Modulation of synaptic plasticity by physiological activation of M1 muscarinic acetylcholine receptors in the mouse hippocampus. Journal of Neuroscience, 25(48):11194-11200.

Skudlarski, P., Jagannathan, K., Calhoun, V. D., Hampson, M., Skudlarska, B. A., and Pearlson, G. (2008). Measuring brain connectivity: diffusion tensor imaging validates resting state temporal correlations. $\mathrm{Neu}$ roimage, $43(3): 554-561$.

Smith, S. M. (2002). Fast robust automated brain extraction. Human Brain Mapping, 17(3):143-155.

Spronk, M., Ji, J. L., Kulkarni, K., Repovs, G., Anticevic, A., and Cole, M. W. (2017). Mapping the human brain's cortical-subcortical functional network organization. bioRxiv.

Stevens, W. D. and Spreng, R. N. (2014). Resting-state functional connectivity MRI reveals active processes central to cognition. Wiley Interdisciplinary Reviews: Cognitive Science, 5(2):233-245.

Swanson, L. and Cowan, W. (1979). The connections of the septal region in the rat. Journal of Comparative Neurology, 186(4):621-655.

Thirion, B., Varoquaux, G., Dohmatob, E., and Poline, J.-B. (2014). Which fMRI clustering gives good brain parcellations? Frontiers in Neuroscience, 8:167.

Unal, C. T., Pare, D., and Zaborszky, L. (2015). Impact of basal forebrain cholinergic inputs on basolateral amygdala neurons. Journal of Neuroscience, 35(2):853-863.

Van Dijk, K. R., Hedden, T., Venkataraman, A., Evans, K. C., Lazar, S. W., and Buckner, R. L. (2010). Intrinsic functional connectivity as a tool for human connectomics: theory, properties, and optimization. Journal of Neurophysiology, 103(1):297-321.

Walt, S. v. d., Colbert, S. C., and Varoquaux, G. (2011). The numpy array: a structure for efficient numerical computation. Computing in Science \& Engineering, 13(2):22-30.

Weissenbacher, A., Kasess, C., Gerstl, F., Lanzenberger, R., Moser, E., and Windischberger, C. (2009). Correlations and anticorrelations in resting-state functional connectivity MRI: a quantitative comparison of preprocessing strategies. NeuroImage, 47(4):1408-1416.

Wenk, G. L. (1997). The nucleus basalis magnocellularis cholinergic system: one hundred years of progress. Neurobiology of Learning and Memory, 67(2):85-95.

Whitehouse, P. J., Price, D. L., Struble, R. G., Clark, A. W., Coyle, J. T., and Delon, M. R. (1982). Alzheimer's disease and senile dementia: loss of neurons in the basal forebrain. Science, 215(4537):12371239.

Yang, C., Thankachan, S., McCarley, R. W., and Brown, R. E. (2017). The menagerie of the basal forebrain: how many (neural) species are there, what do they look like, how do they behave and who talks to whom? Current Opinion in Neurobiology, 44:159-166.

Yeo, B. T., Krienen, F. M., Sepulcre, J., Sabuncu, M. R., Lashkari, D., Hollinshead, M., Roffman, J. L., Smoller, J. W., Zöllei, L., Polimeni, J. R., et al. (2011). The organization of the human cerebral cortex estimated by intrinsic functional connectivity. Journal of Neurophysiology, 106(3):1125-1165.

Zaborszky, L., Csordas, A., Mosca, K., Kim, J., Gielow, M. R., Vadasz, C., and Nadasdy, Z. (2015a). Neurons in the basal forebrain project to the cortex in a complex topographic organization that reflects corticocortical connectivity patterns: an experimental study based on retrograde tracing and 3D reconstruction. Cerebral Cortex, 25(1):118-137. 
Zaborszky, L., Duque, A., Gielow, M., Gombkoto, P., Nadasdy, Z., and Somogyi, J. (2015b). Organization of the basal forebrain cholinergic projection system: specific or diffuse. The Rat Nervous System. Academic Press, San Diego, pages 491-507.

Zaborszky, L., Hoemke, L., Mohlberg, H., Schleicher, A., Amunts, K., and Zilles, K. (2008). Stereotaxic probabilistic maps of the magnocellular cell groups in human basal forebrain. NeuroImage, 42(3):11271141.

Zhang, S., Hu, S., Fucito, L. M., Luo, X., Mazure, C. M., Zaborszky, L., and Chiang-shan, R. L. (2016). Resting-state functional connectivity of the basal nucleus of meynert in cigarette smokers: Dependence level and gender differences. Nicotine ${ }_{3} 3$ Tobacco Research, page ntw209.

Zhang, Y., Brady, M., and Smith, S. (2001). Segmentation of brain mr images through a hidden markov random field model and the expectation-maximization algorithm. IEEE transactions on medical imaging, $20(1): 45-57$.

Zou, K. H., Warfield, S. K., Bharatha, A., Tempany, C. M., Kaus, M. R., Haker, S. J., Wells, W. M., Jolesz, F. A., and Kikinis, R. (2004). Statistical validation of image segmentation quality based on a spatial overlap index. Academic Radiology, 11(2):178-189. 